\title{
Fatores espaciais da ocorrência criminal: modelo estruturador para a análise de evidências empíricas
}

\author{
Environmental factors of the crime occurrence: a framework for the analysis of available \\ evidence
}

Mariana Soares ${ }^{[a]}\left[(\mathbb{D})\right.$, Renato Tibiriçá de Saboya ${ }^{[a]}$

[a] Universidade Federal de Santa Catarina, Florianópolis, SC, Brasil

Como citar: Soares, M., \& Saboya, R. T. (2019). Fatores espaciais da ocorrência criminal: modelo estruturador para a análise de evidências empíricas. urbe. Revista Brasileira de Gestão Urbana, 11, e20170236. https://doi.org/10.1590/21753369.011.001.A010

\section{Resumo}

A grande quantidade de estudos sobre a relação entre os fatores espaciais e a ocorrência de crimes resultou em um quadro de fragmentação que dificulta uma visão integrada de quais são esses fatores e de quão robusta são as evidências sobre a influência de cada um deles, bem como de quais são as múltiplas interações de um mesmo fator em diferentes fases do ato criminal. Para preencher essa lacuna, é proposto um modelo da relação entre o ambiente construído e a ocorrência de crimes que estrutura e explicita essas interações hipotéticas e delineia uma série de fatores intermediadores que ajudam a explicar as conexões causais entre eles e os crimes. 0 modelo propriamente dito é estruturado em três níveis: as quatro etapas da ocorrência criminal (seleção do alvo, aceso ao alvo, execução do crime e fuga), os fatores intermediadores e os aspectos físico-espaciais. Realizou-se uma extensa revisão de literatura em busca de evidências empíricas que permitissem estimar com maior confiança as relações delineadas, e então foram discutidas suas implicações e levantados alguns dilemas sobre conexões causais potencialmente conflitantes reveladas pelo modelo. Como resultados adicionais, apontam-se alguns aspectos sobre os quais as incertezas ainda são significativas, indicando possíveis focos para estudos futuros.

Palavras-chave: Ocorrência de crimes. Ambiente construído. Fatores espaciais. Prevenção ao crime. Desenho urbano.

\section{Abstract}

The large number of studies on the relationship between environmental factors and the occurrence of crimes has resulted in a fragmented state of affairs that makes it hard to establish an integrated view of what these factors are, and how robust the available evidence about each of them is. It is also difficult to establish the different potential interactions that each factor may elicit in different stages of the criminal act. In order to fill this gap, we propose a model of the relationship between built environment and the occurrence of crimes, to structure and unveil these interactions, and outline a series of intermediary factors that help explain the hypothetical causal connections between them. The model is structured in

MS é arquiteta e urbanista, mestre em Arquitetura e Urbanismo, doutora em Arquitetura e Urbanismo, e-mail: mariana_soa@hotmail.com RTS é arquiteto e urbanista, mestre em Planejamento Urbano e Regional, doutor em Engenharia Civil, e-mail: renato.saboya@ufsc.br 
three levels: the four stages of criminal occurrence (target selection, access to target, crime execution and get-away), intermediate factors, and environmental aspects. We carried out an extensive literature review in search of empirical evidence to allow us to estimate with more confidence the proposed relations, and then discussed its implications and indicated some dilemmas about potentially conflicting causal connections revealed by the model. Additionally, we pointed out some aspects about which of the uncertainties are still significant, indicating possible gaps for future studies.

Keywords: Crime occurrence. Built environment. Environmental factors. Crime prevention. Urban design.

\section{Introdução}

Entre os principais desafios a serem enfrentados pelos aglomerados urbanos está, cada vez mais, a segurança que os espaços apropriados e usufruídos pelas pessoas oferecem quanto ao crime. Resultados de Cozens (2011) apontam que, se os cidadãos se sentem seguros, eles se tornam mais propensos a utilizar os espaços, contribuindo para a vitalidade e a sustentabilidade da cidade e do ambiente construído.

Há, como resultado, uma profusão de estudos que investigam a relação entre ações criminosas e características do espaço urbano, utilizando um conjunto de teorias, abordagens, metodologias e recortes temáticos. No entanto, esses estudos encontram-se, atualmente, fragmentados, sendo difícil ter uma noção do conjunto desses fatores explicativos e do nível de confiança sobre a influência de cada um deles nas ocorrências criminais.

Por esses motivos, o conhecimento já existente sobre o tema acaba não se transferindo para a prática do planejamento espacial de cidades e de edificações. Dessa forma, são necessárias a estruturação e a articulação de pesquisas cujos resultados auxiliem planejadores e projetistas a entender como os fatores ambientais podem influenciar a ocorrência de atos criminais, bem como quais são as prováveis consequências de suas escolhas projetuais, permitindo que explorem alternativas e cenários com maiores chances de prevenir a ocorrência de delitos.

Para preencher essa lacuna, propõe-se um modelo cuja intenção é agregar e organizar o que já se sabe sobre a relação entre o ambiente físico e a ocorrência de crimes em uma estrutura lógica, explorando as conexões causais por meio de fatores intermediadores. Adotam-se, para essa estrutura lógica, as diversas etapas do ato criminoso, por proporcionarem um encadeamento compreensível de etapas - ainda que não necessariamente lineares -, que ajudam a entender como os diversos elementos do modelo relacionam-se entre si, bem como tornam mais claros as multiplicidades e os intercruzamentos de linhas de influência que, normalmente, são tratados separadamente na literatura.

Concomitantemente, analisam-se as evidências empíricas disponíveis sobre o quão confiáveis são essas relações, considerando tanto aquelas que as corroboram quanto as que as contradizem, proporcionando, assim, uma ideia mais abrangente do grau de segurança existente na literatura sobre cada uma delas e, ao mesmo tempo, reconhecendo a complexidade do fenômeno sem reduzi-lo a fórmulas prontas e simplificadoras. Pelo fato de as ocorrências criminais estarem muito ligadas a questões culturais e a outros fatores específicos de cada contexto, as conclusões sobre a influência dos fatores espaciais precisam ser vistas com cautela. Entretanto, entende-se que resultados semelhantes em contextos diferentes indicam maior probabilidade de generalização, inclusive para as cidades brasileiras. Nesse sentido, tenta-se também incluir estudos realizados no país, tanto como forma de verificar a validade das explicações para o contexto brasileiro quanto como meio de reforçar ou enfraquecer a confiança nos resultados alcançados em outros países, buscando proporcionar uma visão mais apurada do quão confiáveis são as relações identificadas entre crime e espaço. 0 intuito é revelar maior ou menor nível de confiança sobre essas relações e, com isso, indicar aspectos com maior probabilidade de serem úteis em ações concretas de planejamento. Ao mesmo tempo, as incertezas remanescentes não são evitadas ou vistas como necessariamente problemáticas para o modelo, mas sim como indicativas da necessidade de maiores esforços de pesquisa e oportunidades para novos estudos. 
Entende-se ainda que o estudo dessa relação deve ser parte de iniciativas e de estratégias mais amplas que considerem o crime e suas causas na sua complexidade, incorporando outros aspectos (socioeconômicos, culturais, psicológicos etc.) que são, certamente, mais estruturais e determinantes para sua diminuição.

Foram considerados diversos tipos de crimes, quase todos eles relacionados à noção de oportunidade proporcionada pela situação e pelo espaço, incluindo furtos e roubos a pessoas, edificações (residenciais e não residenciais) e veículos, lesões corporais, vandalismo, homicídio, entre outros. Como cada estudo faz seu próprio recorte quanto aos crimes analisados, indica-se, de maneira mais detalhada, quais são eles quando se apresenta e discute cada evidência empírica considerada.

\section{Condições básicas para a ocorrência criminal}

Para que um crime ocorra, três fatores determinantes precisam se encontrar no espaço e no tempo: um agressor motivado, um alvo adequado e a falta de guardião capaz (Wilcox et al., 2003; Clarke \& Eck, 2003). O papel do espaço é, portanto, central, especialmente para os dois últimos fatores. 0 acesso a um alvo adequado e mesmo a consciência de que ele existe e é possível de ser alcançado são, em grande parte, mediados pelo modo como o espaço está organizado. Da mesma forma, a distribuição de guardiões pelo espaço é grandemente influenciada pela sua organização, pelo modo como diferentes ambientes se relacionam e pela forma como os lugares da cidade se dispõem no tecido urbano. Além disso, os macropadrões cotidianos de deslocamento casa-trabalho, em si mesmos muito influenciados pela configuração da malha urbana, desempenham papel fundamental para a determinação de quem encontra quem, com que frequência e em que circunstâncias. Na Figura 1, são apresentados elementos que podem influenciar cada um desses fatores, que são discutidos com mais detalhes em seguida, com especial atenção para o papel do espaço.

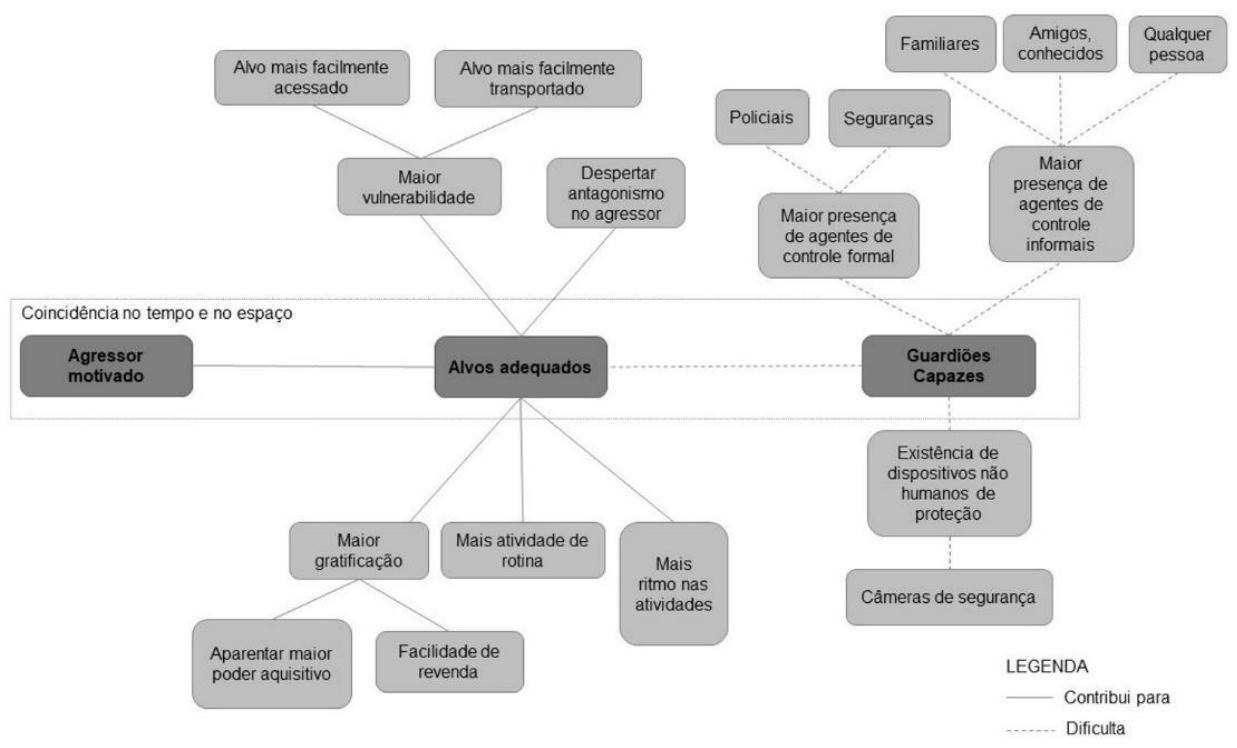

Figura 1 - Mapa conceitual das condições básicas para que um crime ocorra. Fonte: elaboração própria com base em Wilcox et al. (2003).

\section{Agressor motivado}

De uma forma geral, as pessoas cometem atos criminosos para satisfazer seus desejos, na sua maioria motivados pela âmbição de bens materiais e busca por status social (Wilcox et al., 2003). Alguns autores destacam que uma forma de controlar a motivação para o crime é o risco percebido e o 
medo de punição (Gibbs, 1975; Wilcox et al., 2003); portanto, a expectativa de impunidade também pode ser vista como algo positivo para o infrator ao cometer um crime.

Cook (1986), entre outros autores, afirma que o crime é uma escolha racional do criminoso e que suas ações são guiadas pelo princípio do prazer e da dor, na busca de maximizá-lo e de minimizá-la. 0 ambiente em que se encontra e/ou aquele em que se planeja cometer o crime podem aumentar ou diminuir os riscos do infrator, por isso o espaço é um componente importante para uma significativa gama de situações criminosas.

\section{Alvos adequados}

Indivíduos e objetos possuem características que os tornam mais ou menos atraentes para um agressor motivado. Segundo Finkelhor \& Asdigian (1996), a adequação do alvo pode ser dividida em três dimensões fundamentais: a) vulnerabilidade - facilidade e velocidade com que o alvo pode ser acessado e/ou transportado; b) antagonismo - nível de reação de oposição, hostilidade e/ou antipatia que o alvo possa gerar; e c) gratificação - nível que o alvo pode possibilitar de prazer, incluindo aí sua fruição direta ou a facilidade com que possa ser vendido ou usado para adquirir outros bens. Na Figura 2, são apresentados elementos que podem influenciar na avaliação que um agressor faz sobre seu alvo, ao definir um alvo adequado.

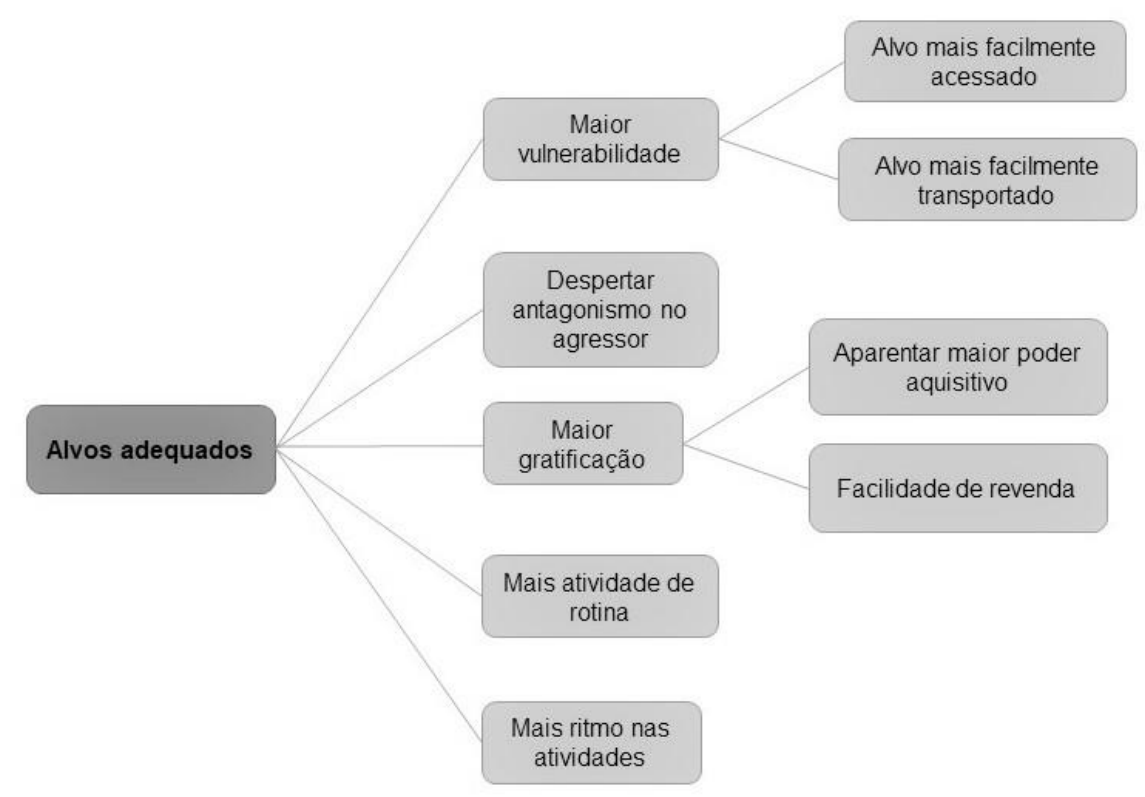

Figura 2 - Mapa conceitual com elementos que podem ser avaliados pelo agressor ao definir um alvo adequado. Fonte: elaboração própria.

Os objetos e as pessoas que constituem bons alvos variam no tempo, no espaço e de acordo com o modelo de crime que se pretende praticar. Uma mesma pessoa pode estar mais ou menos vulnerável aos olhos de um possível infrator em diferentes momentos, por exemplo, dependendo do seu comportamento em determinado instante e do tipo de atenção que presta ao que acontece ao seu redor.

Quanto ao antagonismo, Finkelhor \& Asdigian (1996) observam que os indivíduos ou os objetos podem possuir algumas qualidades ou atributos que aumentam a probabilidade de serem vítimas de um ato criminoso, porque eles podem, sem querer ou sem ter culpa, aumentar o nível de raiva, ciúmes ou outro impulso agressor em determinados infratores. 
Em relação à gratificação, os alvos podem ser percebidos como tendo atributos que poderiam fornecer prazer material ou corporal. O valor monetário associado a pessoas e objetos é a medida mais óbvia do nível de prazer material que ela pode oferecer. Segundo Monteiro (2010), a escolha do local do crime baseia-se, principalmente, na percepção de recompensa e em se estar à procura de vítimas de "qualidade", ou seja, aquelas que pareçam ter o maior poder de compra, pelo tipo de carro ou roupas que usam, lugares que frequentam ou atividades de que participam. Batella \& Diniz (2010) concluíram que ambientes mais prósperos são sinônimos de oportunidades para ação criminosa, uma vez que fornecem mais alvos viáveis e compensadores.

De acordo com Wilcox et al. (2003), as pessoas que apresentam uma atividade de rotina em que passam mais tempo fora de casa, frequentam bares e utilizam transportes públicos regularmente estão mais expostas aos agressores motivados, ou seja, são mais propensas a experimentar vitimizações. Também o ritmo na realização dessas atividades e a sua distribuição pelo tecido urbano podem ser fatores relevantes, uma vez que o ritmo se refere aos padrões de atividade ao longo do tempo e pode deixar o movimento do indivíduo mais previsível, o que poderia torná-lo mais suscetível a uma vitimização (Cohen \& Felson, 1979).

\section{Guardião capaz}

Um guardião capaz é uma pessoa ou um objeto que fornece controle social, podendo exercer tutela e trazer proteção ao alvo (Wilcox et al., 2003). Os indivíduos e os objetos possuem qualidades que se relacionam com laços sociais e controle interpessoal: laços sociais, em nível individual, referem-se ao grau em que os indivíduos, em uma localidade limitada, possuem ligação entre eles e com outras pessoas; já o controle interpessoal no nível individual é o grau em que os indivíduos e os objetos, em um local delimitado, podem ser observados e vigiados por agentes de controle formal (polícia ou segurança), agentes de controle informal (amigos, conhecidos ou qualquer pessoa) e dispositivos não humanos de proteção, por exemplo, câmeras de segurança. Assim, indivíduos e objetos que se encontram mais expostos a uma concentração elevada de guardiões capazes têm menor probabilidade de experimentar eventos criminais, mesmo em um local com elevada quantidade de infratores motivados e de alvos adequados. Conforme aumenta a quantidade de guardiões capazes, aumenta o custo para o infrator, pois o fornecimento de tutela agregada serve como um impedimento ao elevar a chance de o infrator ser identificado, repreendido, impedido e/ou detido. Na Figura 3, são apresentados guardiões capazes que podem diminuir a possibilidade de que um crime ocorra.

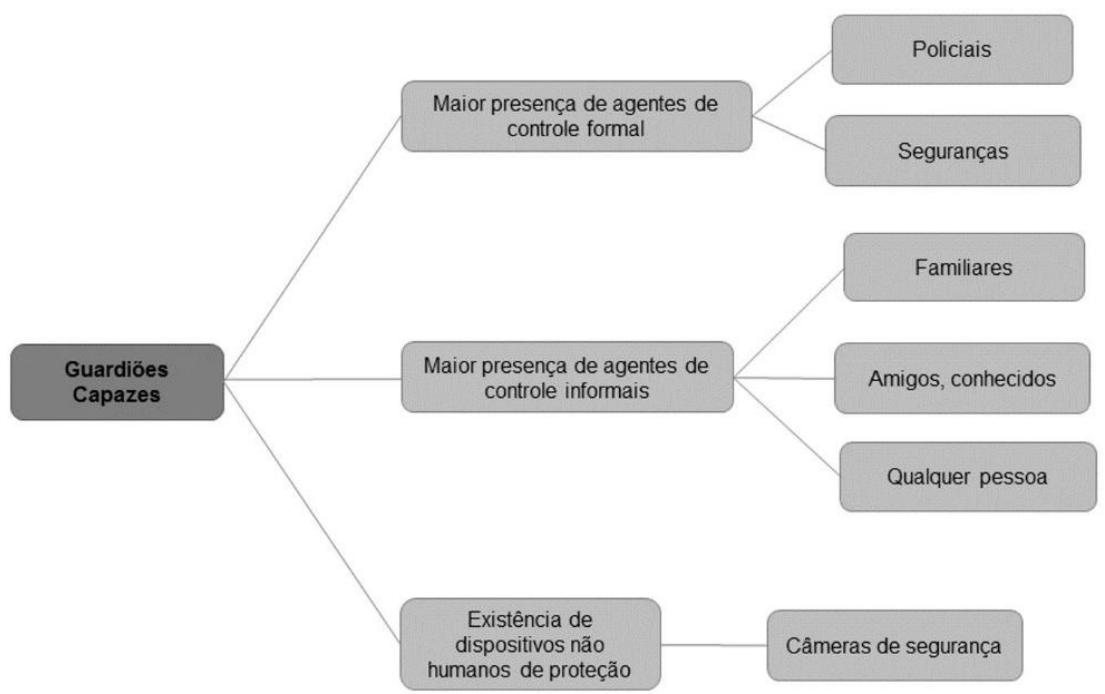

Figura 3 - Mapa conceitual com guardiões capazes que podem diminuir a possibilidade para que um crime ocorra. Fonte: elaboração própria. 


\section{Modelo geral estruturador e as fases da ocorrência criminal}

Ao examinar as teorias e as hipóteses levantadas para explicar a influência do espaço na criminalidade, vê-se que todas elas se referem a uma ou mais etapas segundo as quais as ocorrências acontecem. Entretanto, isso raramente é feito de uma forma sistemática, o que pode dificultar a consideração de que os mesmos fatores espaciais podem atuar em cada uma delas de maneiras diferentes e, às vezes, em direções opostas. Um exemplo disso é a presença de pessoas, o que pode ser um facilitador para a seleção do alvo e o acesso a ele, mas um dificultador para a execução do crime.

Por esse motivo, o modelo foi estruturado segundo quatro etapas da ocorrência criminal: seleção do alvo, acesso ao alvo, execução do crime e fuga. 0 modelo gráfico apresentado na Figura 4 mostra as principais relações entre as fases da ocorrência criminal e os fatores físico-espaciais que nelas podem exercer influência, bem como os conceitos mais abstratos que os intermediam.

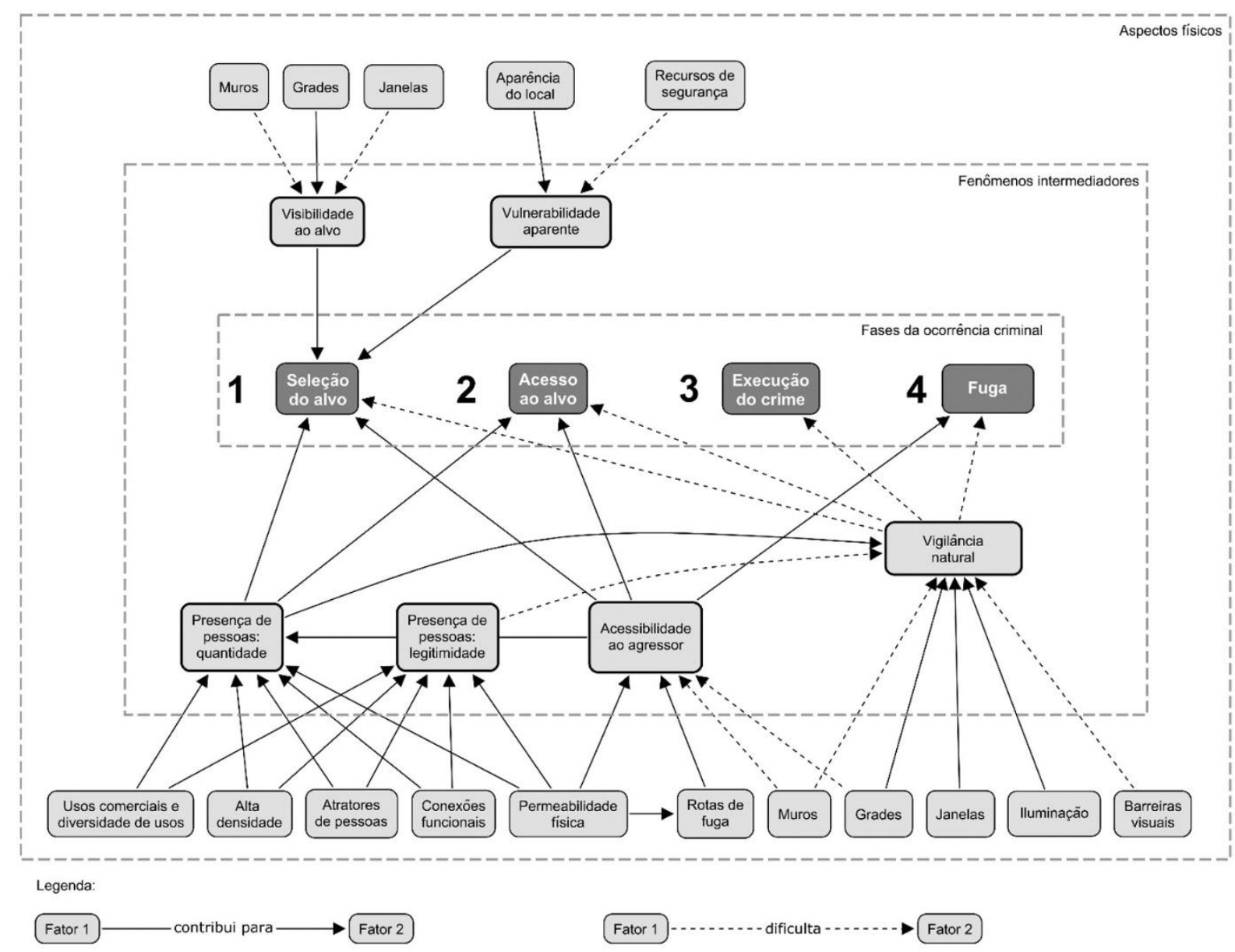

Figura 4 - Mapa conceitual proposto. Fonte: elaboração própria.

\section{Seleção do alvo}

A teoria do padrão de crime defende que os locais de crime não são escolhidos aleatoriamente, possivelmente existindo uma interação entre o espaço de consciência do criminoso (um mapa mental do ambiente) e a distribuição de alvos adequados, embasando a relação entre o infrator, a vítima e o meio em que o crime ocorre (Wortley \& Mazerolle, 2008). Relacionada a isso está a questão das temporalidades das dinâmicas urbanas, em especial os ritmos e os fluxos gerados por diferentes usos do solo, que permitem a construção de diferentes mapas mentais dos ambientes e oportunidades de consciência sobre possíveis alvos. 


\section{Acesso ao alvo}

De acordo com Canter (2003), dois fatores são cruciais para a ligação entre as vítimas e os criminosos: tempo e espaço. As pessoas que cometem crime têm padrões de movimento como todas as outras e circulam em locais que podem ser de sobreposição entre o criminoso e a vítima. 0 criminoso poder acessar o alvo é uma necessidade básica para que o crime ocorra.

\section{Execução do crime}

A etapa de execução do crime corresponde ao momento em que o agressor está efetivamente cometendo o ato. Tal etapa provavelmente seja a fase de maior tensão tanto para a vítima como para 0 infrator, visto que este poderia ser mais facilmente identificado.

\section{Fuga}

Assim que o crime é cometido, o agressor tentará evadir-se do local e colocar-se em segurança. Dependendo do tipo de alvo e do tipo de crime, isso pode envolver fuga em alta velocidade ou comportar-se o mais naturalmente possível, de forma a não despertar atenção. Os primeiros momentos são os mais críticos, nos quais a chance de ser parado ou capturado é maior, e, quanto mais distante do local do crime, menores são os riscos corridos pelo agressor.

\section{Fatores espaciais e as fases da ocorrência criminal}

\section{Visibilidade e vigilância natural}

A possibilidade de ver e de acessar o alvo é essencial para que um crime ocorra, o que, por sua vez, implica que o agressor deva estar próximo ao alvo, isto é, a uma distância curta o suficiente para que ele possa enxergá-lo sem obstáculos (Saboya \& Ribas, 2016). Portanto, o fator visibilidade está diretamente relacionado à acessibilidade para o agressor (o que será tratado mais adiante).

Para poder avaliar a atratividade de um alvo, o criminoso em potencial precisa saber que ele existe e onde ele está. Isso implica quase sempre, para os propósitos deste trabalho, em ver o alvo. Por essa lógica, restringir a visão sobre alvos em potencial tende a ser uma estratégia útil para diminuir as chances de um objeto ser roubado ou furtado. Isso permeia grande parte das decisões tomadas atualmente, por exemplo, os muros altos e opacos que impedem a visão do interior das residências (Caldeira, 2000).

Entretanto, elas nem sempre funcionam. Michael et al. (2001) descobriram que os criminosos observavam os turistas chegando a um parque nos Estados Unidos e preparando-se para sair do veículo. Ao notar seus procedimentos para esconder bens valiosos e a direção em que iniciavam seu percurso, tinham uma ideia do que estava sendo deixado no veículo e do tempo disponível para o furto.

No caso da construção de muros altos e opacos, que não permitem relação com o espaço público e que restringem a amplitude visual entre edificação e espaço público e diminuem a possibilidade de vigilância natural, os resultados também podem não ser os esperados. Em uma residência ou em um edifício com muros altos, o infrator estará protegido dos olhares externos tão logo tenha adentrado o local, dificultando ou mesmo impossibilitando que vizinhos ou passantes vejam o que está acontecendo e intervenham ou chamem auxílio.

Monteiro (1999) observou que espaços mais visíveis tendem a ser mais utilizados do que espaços constituídos por arquiteturas cegas, tais como aqueles constituídos por paredes sem aberturas. 
O mesmo foi encontrado por Moraes et al. (2012) e Saboya et al. (2015) no que diz respeito à relação entre muros cegos e movimento de pedestres. Iannicelli (2009) percebeu que ruas configuradas por muros e paredes cegas apresentam maiores taxas de crimes de furto e roubo em Recife, Pernambuco. Já Saboya et al. (2016) também encontraram indícios dessa associação em Florianópolis, Santa Catarina, para furtos e roubos a pessoas, residências, estabelecimentos e veículos. Quintana (2013) observou a relação da ocorrência de alguns tipos de crime (furto e roubo de residências e de veículos, roubo a pedestres) com a menor existência de visibilidade em áreas com condomínios fechados em Porto Alegre, Rio Grande do Sul. Bondaruk (2007), a partir de entrevistas com detentos, alertou que locais com baixa visibilidade são preferidos para a prática de crimes de homicídio, roubo e furto.

\section{Janelas}

A posição e a quantidade de janelas e de portas visualmente permeáveis dos edifícios, em relação às ruas, aos passeios e ao estacionamentos, entre outras áreas, podem possibilitar uma melhor visualização do espaço público e proporcionar sua supervisão.

Perkins et al. (1993) analisaram 48 quarteirões em Nova Iorque, Estados Unidos, e concluíram que ruas com maior visibilidade das janelas do térreo possuíam menor quantidade de crimes (invasão de propriedade, incêndio, roubo, furto, furto de veículos, lesão corporal, estupro e homicídio). 0 maior número de conexões visuais entre os espaços abertos públicos e as adjacências construídas inibiam, de acordo com o estudo de Zanotto (2002), a ocorrência de furtos, roubos, ameaças, homicídios, lesões corporais, arrombamento e vandalismo, e, segundo a pesquisa de Quintana (2013), de furtos e roubos de residências e veículos e de roubos a pedestres. Vivan \& Saboya (2017) observaram que, em ruas predominantemente residenciais (pelo menos $70 \%$ de residências), a maior quantidade de conexões visuais está relacionada a uma menor quantidade de furtos e roubos a pessoas, residências, estabelecimentos e veículos.

\section{lluminação}

A iluminação é um dos aspectos mais diretamente associados ao risco de crimes, fazendo parte do imaginário popular. Espaços escuros e sombrios são naturalmente associados a lugares perigosos. Isso acontece porque a iluminação pode melhorar ou comprometer a visibilidade de determinado local, ou seja, áreas mais iluminadas permitiriam uma maior facilidade para que transeuntes ou vizinhos percebessem que um crime está acontecendo.

A iluminação (ou falta dela) também pode cumprir um papel relevante na seleção do alvo e no acesso a ele, especialmente quando o crime ocorre no período da noite. Áreas escuras podem servir de proteção aos agressores enquanto estudam o local e planejam o ato.

Painter \& Farrington (1997) observaram que a melhoria da iluminação pública reduziu a quantidade de crimes de roubo, furto, vandalismo e aumentou o uso da rua pelos pedestres. Em revisão sistemática de estudos realizados nos Estados Unidos e no Reino Unido, Farrington \& Welsh (2002) concluíram que a melhoria na iluminação pública reduziu ocorrências criminais de maneira geral em $7 \%$ em oito estudos norte-americanos e em 30\% em cinco estudos ingleses. Zanotto (2002) observou, em estudo em Pelotas, Rio Grande do Sul, que nem sempre a maior iluminação pública garantia a menor ocorrência de furtos, roubos, ameaças, homicídios, lesões corporais, arrombamento e vandalismo, porém encontrou evidências de que as pessoas se sentiam mais seguras em locais bem iluminados.

Cabe notar, entretanto, o aspecto precário da iluminação, tanto no que diz respeito aos crimes em si quanto aos estudos para estabelecer sua relevância. Quando há planejamento antecipado, o criminoso pode, com certa facilidade, quebrar lâmpadas para tornar o local mais escuro e propício para a abordagem a uma pessoa ou a invasão a uma propriedade. No caso dos estudos sobre o tema, nem 
sempre é possível determinar com confiança se as condições de iluminação à época do levantamento são as mesmas de quando o crime ocorreu.

\section{Barreiras visuais}

A falta de amplitude visual pela presença de barreiras visuais e a ausência de intervisibilidade entre as pessoas que estão no mesmo espaço podem impedir a visão de outros indivíduos no momento em que o crime está acontecendo, dificultando a detecção do criminoso e, provavelmente, diminuindo as chances de que o crime seja impedido ou interrompido.

A visibilidade também pode ser importante no sentido de permitir ao potencial agressor saber da existência de um alvo e onde ele se encontra. Assim, a presença de "esconderijos" pode ser uma variável a ser observada pelo infrator no local em que ele pretende cometer o crime, pois esse local pode ser utilizado pelo infrator para criar um elemento surpresa no momento em que ele abordar a vítima, impedindo que ela perceba a sua presença a tempo de evitar o crime. Michael et al. (2001) encontraram que vegetação arbustiva densa era utilizada por criminosos para observar as vítimas em potencial, valendo-se do fato de que essa configuração lhes permitia ver sem serem vistos. Por outro lado, Kuo \& Sullivan (2001) concluíram que a vegetação, em geral, está associada a uma diminuição significativa do número de roubos a pessoas e que, mais especificamente, extratos vegetais que não obstruem a visão, tais como grama e árvores altas e espaçadas, não aumentam a criminalidade.

\section{Acessibilidade ao agressor}

A acessibilidade ao agressor, assim como a visibilidade ao alvo, é um dos fatores mais básicos intermediando a forma do ambiente e a ocorrência de crimes. Em algumas situações, elas aparecem conjugadas, como é o caso dos muros, mas conceitualmente vale a pena tratá-las separadamente, porque seus efeitos são diferentes. No caso de grades, por exemplo, a visibilidade é preservada (tanto para o agressor quanto para os demais usuários do local, que podem exercer vigilância), enquanto o acesso é dificultado.

Com relação ao acesso ao alvo antes e durante a execução do crime, essa relação é bem conhecida e pouco controversa. Dela decorrem uma série de dispositivos e de providências que tem sido tomada para evitar o crime desde há muito tempo, tais como portas, cadeados, grades nas janelas, muros altos, cercas elétricas, elementos pontiagudos etc. 0 acesso ao criminoso após a execução do crime merece maior atenção e será tratado mais adiante, no item sobre permeabilidade e acessibilidade em geral.

\section{Vulnerabilidade aparente}

\section{Aparência do local}

A deterioração física e os sinais de negligências (pichações, lixo etc.) podem afetar a percepção do criminoso sobre o controle social, fazendo-os perceber essas áreas como vulneráveis. Wilson \& Kelling (1982), Sousa \& Kelling (2006) e Wortley \& Mazerolle (2008) argumentam que esses fatores podem trazer uma sensação de desordem e falta de controles sociais que tendem a levar à ocorrência de crimes mais graves.

Hope \& Hough (1988) e Xu et al. (2005) observaram que áreas que apresentam sinais de deterioração física e desordem podem ser evitadas por seus usuários, uma vez que estão diretamente relacionadas com a sensação de medo e de insegurança. Nessas condições, um círculo vicioso pode ser criado, visto que a deterioração é um efeito da falta de controle social e/ou apropriação do lugar 
(responsáveis pelo local, usuários e moradores deixam de cuidar e fazer manutenção); por causa disso, usuários são afastados e têm orgulho e disposição em cuidar do lugar diminuídos, tornando-se locais cada vez menos utilizados e mais perigosos.

Harcourt \& Ludwig (2006) avaliaram a teoria das "janelas quebradas" e a forma de policiamento conhecida como "tolerância zero". Em síntese, a análise realizada por eles não encontrou resultados empíricos que evidenciem que o combate aos crimes menores (pichação, vandalismo etc.) e à desordem leve claramente a uma redução de crime mais sérios.

\section{Vigilância mecanizada e recursos de segurança}

A presença de recursos de segurança, ou seja, agentes formais (policiais e seguranças) e dispositivos de proteção, como câmeras, pode ser interpretada pelo agressor como um aumento no risco de ser detectado quando estuda o local, no momento em que comete o crime, durante a fuga ou até mesmo depois disso, uma vez que, no caso das câmeras, os vídeos podem levar à sua posterior identificação e apreensão.

Um estudo da National Association of Convenience Stores (1991) realizado em 340 lojas de varejo nos Estados Unidos descobriu que os roubos diminuíram em lojas que mudaram de um para dois funcionários, enquanto estudos com patrulhas de guardas de segurança móvel no metrô de Londres, Inglaterra, e em estações ferroviárias na América do Norte mostraram que a presença desses agentes não reduziu os roubos nesses locais (Webb \& Laycock, 1992; Kenney, 1986).

0 uso de câmeras de segurança, de uma forma geral, tem aparecido como um fator importante para a redução de crimes de violência, drogas, furto e roubo de propriedades e de veículos, roubo a transeuntes, dano patrimonial, fraude e manipulação de bens roubados (Armitage et al., 1999; Brown, 1995; Clarke et al., 1991; Poyner, 1991). Resultados de estudos mostrados por Cozens et al. (2005) reforçaram o entendimento de que câmeras podem dissuadir os crimes de roubo a pessoas e de furto e roubo de veículos, devido ao aumento do risco de o infrator ser detectado.

Farrington \& Welsh (2002) realizaram uma revisão sistemática do impacto da prevenção de crimes com uso de câmeras de segurança e observaram um efeito significativo na redução dos crimes de furto e roubo de veículos em oito estudos. Entretanto, nenhum efeito foi encontrado em relação a crimes violentos (a partir de cinco estudos).

\section{Presença de pessoas: quantidade e legitimidade}

A quantidade de pessoas transitando pelo local é uma variável importante na seleção do alvo, no acesso a ele, na execução do crime e no momento da fuga, ou seja, em todas as fases da ocorrência criminal. Em grande parte, ela é mediada pela vigilância natural em sua influência no crime, mas isso nem sempre é necessariamente o caso, sobretudo nas duas primeiras fases.

Pesquisas na perspectiva da geografia comportamental observaram que lugares com os quais os infratores são mais familiarizados, ou seja, locais próximos de onde eles moram, trabalham, recreiam-se ou passam constantemente, são mais propícios a serem escolhidos para os crimes (Brantingham \& Brantingham, 1981; Rengert \& Wasilchick, 1985).

Por outro lado, locais com grande quantidade de pessoas possuem maior capacidade de exercer vigilância natural que áreas mais desertas. Especialmente no momento de execução do crime, a presença de pessoas representa um risco ao agressor, assim como as etapas de seleção do alvo e de acesso a ele.

A possibilidade de cruzar com mais pessoas durante a fuga também aumentaria a possibilidade de ser perseguido e de ser barrado por alguém, aumentando o risco de ser pego. No entanto, dependendo do contexto, um maior número de pessoas poderia servir de escudo para o criminoso sumir facilmente na multidão. 
Sorensen (2003), por outro lado, levantou a questão de que a quantidade de espectadores não está diretamente relacionada à probabilidade de intervenção, alegando que, em áreas frequentadas apenas por pessoas que não se conhecem e não possuem um senso de comunidade, as taxas de furtos de residências podem ser altas. No entanto, assinala-se que a possibilidade de ser identificado e/ou detido é sempre um fato a ser considerado pelo infrator, mesmo nessas situações de anonimato, pois a incerteza quanto às reações ainda permanece.

Outro fator importante é a legitimidade da presença das pessoas no local, em grande medida relacionada à quantidade de pessoas, uma vez que, quanto mais gente, maior tende a ser o anonimato entre os usuários. A diversidade de usos do solo, mais especificamente os usos não residenciais, bem como a existência de pontos de atração de diferentes naturezas, fornece a justificativa para a presença de qualquer pessoa.

Cabe destacar, entretanto, que Bondaruk (2007), a partir da análise de entrevistas com criminosos em Curitiba, Paraná, concluiu que o menor fluxo de pessoas é um indicador importante para o criminoso na escolha de locais para a prática de homicídio, roubo e furto. Assim, existe uma dupla possibilidade em relação à quantidade de pessoas nos locais em que ocorrem crimes: se, por um lado, mais pessoas proporcionam mais alvos e dão maior legitimidade à presença de potenciais transgressores, por outro proporcionam maior vigilância informal, aumentando o risco para eles.

\section{Permeabilidade física}

As barreiras físicas ao movimento permitem maior ou menor permeabilidade e acessibilidade a determinada área, provavelmente influenciando na movimentação de pessoas no espaço em estudo e também na vigilância natural do local. A posição das barreiras físicas ao movimento define o posicionamento das passagens e, em consequência disso, interfere no sistema de percursos que permeia e nutre os espaços.

As condições ambientais também podem influenciar ao ser avaliada pelo infrator a possibilidade de fuga, uma vez que a permeabilidade física pode facilitá-la ou dificultá-la. Tecidos desconectados e com mais quebras tendem a ser mais favoráveis apenas quando ele conhece bem o local. Assim, alguns autores defendem que locais com maior permeabilidade podem ser mais buscados por infratores para a prática de crimes contra a propriedade (Poyner, 1983; Taylor, 2002).

Ainda não existe um consenso entre autores sobre a maior permeabilidade ser positiva ou negativa para que haja ocorrências criminais. Assim, embora o modelo que favorece a permeabilidade, gerando encontro entre pessoas e aumentando os "olhos" nas ruas, seja defendido por um grande número de autores (entre eles, Jacobs, 1961; Dovey, 1998; Hillier \& Shu, 2000; Moraes \& Jelvez, 2007; Dempsey, 2008), há outros autores que encontraram o contrário em seus estudos (entre eles, Ycaza, 1992; Lasley, 1996; Zanotto, 2002; Taylor, 2002; Yang, 2006; Hillier \& Sahbaz, 2012; Quintana, 2013; Vivan \& Saboya, 2017).

Hillier \& Sahbaz (2012) estudaram esse aspecto em mais detalhes e concluíram que vias integradas no tecido na escala da cidade como um todo tendem a possuir maiores taxas de furtos e roubos de residências, enquanto aquelas integradas na escala local (do bairro ou da vizinhança) tendem a ser mais seguras. Monteiro \& Cavalcanti (2017), por outro lado, encontraram resultados opostos: seus estudos indicaram que vias mais integradas localmente estão associadas a maiores quantidades de roubos a transeuntes, o que, entretanto, não indica como seria a relação se fosse considerada a taxa de crimes em vez de quantidades brutas.

É importante ressaltar também a possível diferença na influência sobre diferentes tipos de crime exercida pela permeabilidade. Estudos, como os de Hillier \& Shu (1999) e Hillier \& Sahbaz (2012), alertam que pequenos furtos, por exemplo, o de batedores de carteira, tendem a ocorrer em ruas com maior movimento de pessoas, nas quais sua ação pode passar facilmente despercebida, enquanto crimes violentos seriam mais frequentes em espaços menos acessíveis, nos quais a presença de pessoas tende a ser menor. Van Nes \& López (2010) observaram que vias com maior movimento de veículos seriam mais visadas para roubo e furto de veículos por apresentarem maior número de alvos em 
potencial. Já para furto e roubo de residências, ruas com menor movimento de pessoas são as preferidas, mas ruas residenciais segregadas localmente podem ser lugares seguros quando estão integradas globalmente dentro do sistema urbano (Hillier, 2004).

\section{Usos comerciais e diversidade de usos}

A variedade de uso do solo, embora possa trazer uma maior movimentação de pessoas e, assim, mais "olhos na rua", ao propiciar e legitimar a existência de mais pessoas, pode trazer também maior quantidade de infratores. Dessa forma, alguns autores defendem que a variedade de uso do solo é um fator importante para a redução da criminalidade (Jacobs, 1961; Burton \& Mitchell, 2006; Moraes \& Jelvez, 2007), enquanto outros acreditam que alguns usos e a maior quantidade de movimento de pessoas, gerada pelos diferentes usos do solo, podem atrair infratores (Perkins et al., 1993; Taylor \& Harrell, 1996; Yang, 2006; Schneider \& Kitchen, 2007; Anderson et al., 2013; Saboya et al., 2016).

Taylor \& Harrell (1996) revisaram uma grande quantidade de estudos empíricos e concluíram que moradores de locais com usos não residenciais são menos propensos a intervir se virem algo suspeito e apresentam maiores taxas de vitimização. Carpaneda (2008) identificou que a superquadra de Brasília, Distrito Federal, com maior número de furtos, roubos e vandalismo possuía diversidade de comércio e que a superquadra com menos ocorrências criminais era composta por comércio local pouco diversificado e atrativo. Saboya et al. (2016) constataram que os locais de crimes (roubos e furtos a pessoas, residências, estabelecimentos e veículos) possuíam, em média, maiores proporções de comércio e diversidade de usos do solo. Monteiro \& Cavalcanti (2017) também encontraram associação entre diversidade de usos e quantidades de roubos a transeuntes.

Hillier \& Sahbaz (2012) observaram que, em ruas com comércio varejista, o número de roubos a pedestres aumentava, em média, 2,4 vezes. Entretanto, a quantidade de pedestres nesses locais era 4 vezes maior do que em ruas residenciais, o que demonstra que o aumento no número de crimes não foi proporcional ao aumento do número de alvos em potencial.

Em síntese, um exame da literatura com relação aos usos do solo deixa claro que as evidências de que usos comerciais estão associados a uma maior taxa de ocorrências de crimes são muito mais sólidas e numerosas do que o contrário, ainda que, aparentemente, isso esteja mais relacionado aos crimes que acontecem nas edificações. Para os roubos a pedestres, quando considerada a taxa de furtos e roubos (em oposição à quantidade bruta), as evidências sugerem que a chance de ser vítima desse crime é menor em ruas comerciais.

\section{Densidade populacional e de atividades}

As densidades populacionais incorrem nos mesmos possíveis efeitos contraditórios da quantidade de pessoas: por um lado, podem facilitar aos agressores identificar e acessar seus alvos; por outro, a vigilância natural tende a ser maior em áreas mais densas. Além disso, maiores densidades tendem a aumentar exponencialmente a quantidade de contatos não planejados entre vítimas e criminosos potenciais, o que teria como efeito um aumento na taxa de crimes.

Watts (1931), em um estudo de condados no Canadá nas décadas de 1910 e 1920, encontrou taxas de condenações penais de 500 para 10 mil habitantes em regiões densas e de apenas 90 em regiões menos densas, concluindo que a densidade populacional seria um fator explicativo para o crime. Kvalseth (1977), por outro lado, examinou cinco estudos empíricos sobre os possíveis efeitos da densidade populacional e dos fenômenos correlatos (tamanho populacional e taxa de crescimento) sobre homicídios, estupros, lesões corporais e roubos e observou que nenhum deles encontrou relações positivas quanto à densidade; aliás, um deles encontrou justamente o contrário, com significância estatística para crimes contra a propriedade. Mais recentemente, Battin \& Crowl (2017) obtiveram o mesmo resultado, encontrando associação negativa entre a densidade populacional e o crime (todos os reportados oficialmente no período considerado), mesmo controlando outros fatores relevantes, como 
nível de pobreza, porcentagem de mulheres responsáveis pelo domicílio e edificações vazias. Barause (2017) constatou que, à medida que diminuíam o número médio de pavimentos e a densidade linear de economias, também diminuíam as taxas de roubos, furtos, desordem, crimes contra a vida e crimes relacionados às drogas em Florianópolis, Santa Catarina.

Portanto, apesar de haver alguma discordância entre os resultados alcançados por diferentes autores, parece haver uma maior tendência à conclusão de que não existe relação entre densidade populacional e crime, ou que a primeira está associada, na verdade, a menores taxas do segundo.

\section{Atratores de pessoas}

Wilcox et al. (2003) relatam que escolas e locais de trabalho possibilitam um elevado tráfego de não residentes (estudantes ou empregados do local), aumentando, dessa forma, a oportunidade para atos criminosos. A existência de atratores que gerem uma maior circulação de pessoas pode significar mais alvos e oportunidades para o crime, assim como maior possibilidade de potenciais transgressores conhecerem o local, familiarizarem-se com ele e identificarem alvos (Cozens, 2011).

\section{Conexões funcionais (portas)}

As conexões funcionais, ou seja, as portas que permitem a ligação entre o interior e o exterior das edificações, podem influenciar na maior movimentação de pessoas pelo local. Alguns autores observaram a relação do maior número de conexões funcionais e espaços mais seguros (Hillier \& Hanson, 1984; Hillier \& Shu, 2000; Shu, 2000), enquanto outros não perceberam relações entre esses elementos (Vivan \& Saboya, 2017) ou encontraram relação positiva entre a quantidade de portas e a ocorrência de roubos e furtos a pessoas, residências, estabelecimentos e veículos (Saboya et al., 2016; Monteiro \& Cavalcanti, 2017), possivelmente porque as portas estão associadas a áreas com maior incidência de usos não residenciais e também porque representam possíveis pontos de entrada na propriedade e, portanto, são elementos potencialmente vulneráveis.

\section{Considerações finais}

O modelo apresentado oferece uma estrutura para o entendimento das variáveis do ambiente construído que exercem alguma influência sobre a ocorrência criminal, tanto em nível urbano quanto de edificação. Buscou-se conferir a essa estrutura uma lógica que facilitasse seu entendimento e o mapeamento de eventuais lacunas que pudessem ser objeto de estudos futuros.

O exame das evidências empíricas, apesar de não possuir ambição de exaustividade, permitiu uma avaliação robusta da confiabilidade das relações de influência delineadas, bem como uma identificação daqueles aspectos em que ainda há maior discordância quanto aos resultados ou que merecem tratamentos teóricos mais cuidadosos. Na medida do possível, tentou-se incluir referências empíricas desenvolvidas no Brasil, tendo em vista que as conclusões alcançadas em estudos na Europa e nos Estados Unidos, que concentram a maior parte dessa produção, não são necessariamente transferidas diretamente para as cidades brasileiras, as quais possuem realidades socioeconômicas e urbanas bastante diferentes.

O modelo proposto permitiu o confronto e o tensionamento entre linhas de (suposta) causalidade que estavam dispersas e fragmentadas em estudos independentes, colocando-as frente a frente. Isso, por sua vez, permitiu uma visão mais equilibrada dos fatores e de como eles podem funcionar de maneira diferente, dependendo da etapa em que o ato criminoso se encontra. Além disso, essa explicitação dos pontos de conflito entre hipóteses permitiu articular novos entendimentos sobre o 
fenômeno e suas ramificações, especialmente por meio da explicitação dos tipos de crime para os quais foram encontradas evidências empíricas de associação com variáveis do ambiente construído.

A visão mais abrangente e estruturada proposta nesse modelo, incorporando as fases da ocorrência criminal, os fenômenos intermediadores relacionados a elas e como esses fenômenos podem ser influenciados por aspectos físicos do ambiente, é o ponto que deve ser mais bem compreendido por planejadores e arquitetos como forma de obter uma melhor estimativa sobre como suas decisões projetuais podem afetar ou beneficiar determinados comportamentos e situações vivenciadas nos espaços relacionadas à ocorrência de crimes. Mais especificamente, um desdobramento desejável para este trabalho é a consideração desses aspectos nas discussões e nas decisões relativas às normas de uso e ocupação do solo, em várias frentes: os limites à ocupação (densidades construtivas e populacionais), possíveis diretrizes para a forma edificada, atualmente virtualmente inexistentes nos planos diretores (visibilidade entre edificação e passeios, previsão de janelas e portas), definição das atividades permitidas e proibidas em cada zona (uso do solo) e normas para a criação de novos parcelamentos (permeabilidade física). Tudo isso, obviamente, deve ser confrontado com outros critérios de desempenho além da segurança, de modo que o resultado final faça jus à importância desse item contextualizado com os demais desejos e as necessidades urbanas.

\section{Agradecimentos}

Esta pesquisa contou com o apoio do Conselho Nacional de Desenvolvimento Científico e Tecnológico (CNPq), por meio de financiamento de projeto de pesquisa, e da Fundação de Amparo à Pesquisa do Estado de Santa Catarina (Fapesc), mediante financiamento parcial de bolsa de doutorado.

\section{Referências}

Anderson, J., MacDonald, J., Bluthenthal, R., \& Ashwood, J. S. (2013). Reducing crime by shaping the built environment with zoning: an empirical study of Los Angeles. University of Pennsylvania Law Review, 161(3), 699-756.

Armitage, R., Smyth, G., \& Pease, K. B. (1999). CCTV evaluation. In K. Painter, \& N. Tilley (Eds.), Surveillance of public space: CCTV, street lighting and crime prevention (Crime Prevention Studies, vol. 10). Monsey: Criminal Justice Press.

Barause, L. (2017). Espaço urbano, uso do solo e criminalidade: Forma da cidade e ocorrência de crimes na Área Conurbada de Florianópolis (Dissertação de Mestrado). Universidade Federal de Santa Catarina, Florianópolis.

Batella, W. B., \& Diniz, A. M. A. (2010). Análise espacial dos condicionantes da criminalidade violenta no estado de Minas Gerais. Sociedade \& Natureza, 22(1), 151-163. http://dx.doi.org/10.1590/S1982-45132010000100011.

Battin, J. R., \& Crowl, J. N. (2017). Urban sprawl, population density, and crime: an examination of contemporary migration trends and crime in suburban and rural neighborhoods. Crime Prevention and Community Safety, 19(2), 136150. http://dx.doi.org/10.1057/s41300-017-0020-9.

Bondaruk, R. L. (2007). A prevenção do crime através do desenho urbano. Curitiba: Edição do Autor.

Brantingham, P. J., \& Brantingham, P. L. (1981). Environmental criminology. Beverly Hills: Sage Publications.

Brown, B. (1995). CCTV in town centres: three case studies (Police Research Group Crime Detection and Prevention Series Paper, no. 68). London: HMSO.

Burton, E., \& Mitchell, L. (2006). Inclusive urban design: streets for life. Oxford: Architectural Press.

Caldeira, T. P. R. (2000). Cidade de muros: crime, segregação e cidadania em São Paulo (1. ed). São Paulo: Editora 34.

Canter, D. (2003). Mapping murder: the secrets of geographical profiling. London: Virgin Books. 
Carpaneda, L. V. (2008). Contribuições para o desenho de espaços seguros: um estudo de caso nas superquadras do plano piloto de Brasília (Dissertação de Mestrado). Faculdade de Arquitetura e Urbanismo da Universidade de Brasília, Brasília.

Clarke, R. V., \& Eck, J. (2003). Become a problem-solving crime analyst: in 55 small steps. London: Jill Dando Institute of Crime Science.

Clarke, R. V., Field, S., \& Mcgrath, G. (1991). Target hardening of banks in Australia and displacement of robberies. Security Journal, 2(1), 84-90.

Cohen, L. E., \& Felson, M. (1979). Social change and crime rate trends: a routine activity approach. American Sociological Review, 44(4), 588-608. http://dx.doi.org/10.2307/2094589.

Cook, P. J. (1986). The demand and supply of criminal opportunities. Crime and Justice, 7, 1-27.

Cozens, P. M. (2011). Urban planning and environmental criminology: towards a new perspective for safer cities. Planning Practice and Research, 26(4), 481-508. http://dx.doi.org/10.1080/02697459.2011.582357.

Cozens, P. M., Saville, G., \& Hillier, D. (2005). Crime prevention through environmental design (CPTED): a review and modern bibliography. Property Management, 23(5), 328-356. http://dx.doi.org/10.1108/02637470510631483.

Dempsey, N. (2008). Quality of the built environment in urban neighbourhoods. Planning Practice and Research, 23(2), 249-264. http://dx.doi.org/10.1080/02697450802327198.

Dovey, K. (1998). Safety and danger in urban design. In Conference Safer Communities: Strategic Directions in Urban Planning (pp. 1-8). Melbourne: Australian Institute of Criminology \& Victorian Community Council Against Violence.

Farrington, D. P., \& Welsh, C. (2002). Effects of improved street lighting on crime: a systematic review (Home Office Research Study, no. 251). London: Research and Statistics Directorate and predecessors: British Crime Survey Datasets.

Finkelhor, D., \& Asdigian, N. J. (1996). Risk factors for youth victimization: beyond a lifestyles/routine activities theory approach. Violence and Victims, 11(1), 3-19. http://dx.doi.org/10.1891/0886-6708.11.1.3. PMid:8870212.

Gibbs, J. P. (1975). Crime, punishment, and deterrence. New York: Elsevier.

Harcourt, B. E., \& Ludwig, J. (2006). Broken windows: new evidence from new york city and a five-city social experiment. The University of Chicago Law Review, 73(1), 271-320.

Hillier, B. (2004). Can streets be made safe? Urban Design International, 9(1), 31-45.

http://dx.doi.org/10.1057/palgrave.udi.9000079.

Hillier, B., \& Hanson, J. (1984). The social logic of space. Cambridge: Cambridge University Press. http://dx.doi.org/10.1017/CB09780511597237.

Hillier, B., \& Sahbaz, O. (2012). Safety in numbers: high-resolution analysis of crime in street networks. In V. Ceccato (Ed.), The urban fabric of crime and fear. Dordrecht: Springer Netherlands.

Hillier, B., \& Shu, S. (1999). Do burglars understand defensible space? New evidence on the relation between crime and space. Inglaterra: Space Syntax Laboratory.

Hillier, B., \& Shu, S. (2000). Crime and urban layout: the need for evidence. In S. Ballantyne, V. MacLaren, \& K. Pease (Eds.), Secure foundations: key issues in crime prevention, crime reduction and community safety (pp. 224-248). London: University College London.

Hope, T., \& Hough, M. (1988). Area crime, and incivility: a profile from the british crime survey. In T. Hope, \& M. Shaw (Eds.), Communities and crime reduction (pp. 30-47.). London: HMSO.

Iannicelli, A. C. P. (2009). Arquitetura e criminalidade: uma análise sobre o padrão de crime no bairro de Boa Viagem. Recuperado em 15 de junho de 2015, de http://www.youblisher.com/p/52408-ARQUITETURA-E-CRIMINALIDADEuma-analise-sobre-o-padrao-de-crime-no-bairro-de-b/

Jacobs, J. (1961). The death and life of great American cities. New York: Vintage Books.

Kenney, D. (1986). Crime on the subways: measuring the effectiveness of the guardian angels. Justice Quarterly, 3(4), 481496. http://dx.doi.org/10.1080/07418828600089071. 
Kuo, F. E., \& Sullivan, W. C. (2001). Environment and crime in the inner city does vegetation reduce crime? Environment and Behavior, 33(3), 343-367.

Kvalseth, J. O. (1977). A note on the effects of population density and unemployment on urban crime. Criminology, 15(1), 105-110. http://dx.doi.org/10.1111/j.1745-9125.1977.tb00051.x.

Lasley, J. R. (1996). Using traffic barriers to design out crime: a program evaluation of lapd's operation cul-de-sac. Fullerton: National Institute of Justice, California State University.

Michael, S. E., Hull, R. B., \& Zahm, D. L. (2001). Environmental factors influencing auto burglary: a case study. Environment and Behavior, 33(3), 368-388. http://dx.doi.org/10.1177/00139160121973034.

Monteiro, C. (2010). Spatial analysis of street crime. In S. G. Shoham, P. Knepper, \& M. Kett (Eds.), International handbook of criminology (pp. 619-648). Boca Raton: CRC Press. http://dx.doi.org/10.1201/9781420085525-c22.

Monteiro, C., \& Cavalcanti, R. (2017). Perfis espaciais urbanos para avaliação de lugares vulneráveis ao crime. In V. Moraes, No, R. T. Saboya, \& T. Carvalho (Eds.), Efeitos da Arquitetura: os impactos da urbanização contemporânea no Brasil (pp. 137-161). Brasília: FRBH.

Monteiro, P. M. M. (1999). Espaços livres públicos de São Cristóvão - padrões espaciais e sociais. In Anais do $8^{\circ}$ ANPUR. Porto Alegre: ANPUR.

Moraes, V. No., \& Jelvez, J. A. Q. (2007). O espaço urbano como dimensão ativa na incidência do crime. Recuperado em 15 de junho de 2015, de http://urbanismo.arq.br/metropolis/wp-content/uploads/2009/05/espaco-urbano-e-adistribuicao-do-crime-netto-e-jelvez-2007.pdf

Moraes, V. No., Vargas, J. C., \& Saboya, R. T. (2012). (Buscando) os efeitos sociais da morfologia arquitetônica. Urbe. Revista Brasileira de Gestão Urbana, 4(446), 261-282. http://dx.doi.org/10.7213/urbe.7400.

National Association of Convenience Stores. (1991). Convenience store security report and recommendations. Alexandria: National Association of Convenience Stores.

Painter, K. A., \& Farrington, D. P. (1997) The crime reducing effect of improved street lighting: the dudley project. In R. V. Clarke (Ed.), Situational crime prevention: successful case studies (2nd. ed., pp. 209-226). Guilderland: Harrow and Heston.

Perkins, D. D., Wandersman, A., Rich, R. C., \& Taylor, R. B. (1993). The physical environment of street crime: Defensible space, territoriality and incivilities. Journal of Environmental Psychology, 13(1), 29-49. http://dx.doi.org/10.1016/S02724944(05)80213-0.

Poyner, B. (1983). Designing against crime: beyond defensible space. London: Butterworths.

Poyner, B. (1991). Situational crime prevention in two parking facilities. Security Journal, 2(1), 96-101.

Quintana, E. B. (2013). Influência de características físico-espaciais na ocorrência de crimes e na percepção de segurança em áreas residenciais com condomínios fechados (Dissertação de Mestrado). Programa de Pós-Graduação em Planejamento Urbano e Regional da Universidade Federal do Rio Grande do Sul, Porto Alegre.

Rengert, G., \& Wasilchick, J. (1985). Suburban burglary. Springfield: Charles C. Thomas.

Saboya, R., \& Ribas, L. (2016). Criminalidade, uso do solo e forma do ambiente construído: Investigando a influência da visibilidade, acessibilidade e diversidade na distribuição de ocorrências criminais (Relatório de Pesquisa). Florianópolis: UFSC.

Saboya, R., Banki, G. H., \& Santana, J. M. A. (2016). Uso do solo, visibilidade e ocorrência de crimes: um estudo de caso em Florianópolis, Santa Catarina. Oculum Ensaios, 13(2), 255-274. http://dx.doi.org/10.24220/2318-0919v13n2a2990.

Saboya, R., Moraes, V. M. No., \& Vargas, J. C. (2015). Fatores morfológicos da vitalidade urbana: uma investigação sobre o tipo arquitetônico e seus efeitos. Arquitextos - Vitruvius, 15(180.02). Recuperado em 15 de junho de 2015, de http://vitruvius.com.br/revistas/read/arquitextos/15.180/5554

Schneider, R., \& Kitchen, T. (2007). Crime prevention and the built environment. London: Routledge. 
Shu, S. (2000). Housing layout and crime vulnerability (PhD thesis). Bartlett School of Graduate Studies, University College, London.

Sorensen, D. (2003). The nature and prevention of residential burglary: a review of the international literature with an eye towards prevention in Denmark. Recuperado em 15 de junho de 2015, de

http://www.justitsministerietdk/sites/default/files/media/Arbejdsomraader/Forskning/Forskningspuljen/2011/200 3/The_Nature_and_Prevention_of_Residential_Burglary.pdf

Sousa, W. H., \& Kelling, G. L. (2006). Of broken windows, criminology and criminal justice. In D. Weisburd, \& A. Braga (Eds.), Police innovation: contrasting perspectives (pp. 77-97). Cambridge: Cambridge University Press.

Taylor, R. B. (2002). Crime Prevention through Environmental Design (CPTED): yes, no, maybe, unknowable, and all of the above. In R. B. Bechtel, \& A. Churchman (Eds.), Handbook of environmental psychology (pp. 413-426). New York: J. Wiley \& Sons.

Taylor, R., \& Harrell, A. (1996). Physical environment and crime. Washington: National Institute of Justice, US Department of Justice.

Van Nes, A., \& López, M. (2010). Macro and micro scale spatial variables and the distribution of residential burglaries and theft from cars: an investigation of space and crime in the Dutch cities of Alkmaar and Gouda. The Journal of Space Syntax, $1(2), 296-314$

Vivan, M., \& Saboya, R. T. (2017). Arquitetura, espaço urbano e criminalidade: efeitos da visibilidade na distribuição da ocorrência de crimes. In V. Moraes, No, R. T. Saboya, \& T. Carvalho (Eds.), Efeitos da Arquitetura: os impactos da urbanização contemporânea no Brasil (pp. 163-182). Brasília: FRBH.

Watts, R. E. (1931). The influence of population density on crime. Journal of the American Statistical Association, 26(173), 11-20. http://dx.doi.org/10.1080/01621459.1931.10503145.

Webb, B., \& Laycock, G. (1992). Reducing crime on the london underground: an evaluation of three pilot projects (Crime Prevention Unit Paper, no. 30). London: HMSO.

Wilcox, P., Land, K. C., \& Hunt, S. A. (2003). Criminal circumstance: a dynamic multi-contextual criminal opportunity theory. New York: Walter de Gruyter.

Wilson, J., \& Kelling, G. (1982). Broken windows: the police and neighbourhood safety. Magazine Atlantic Monthly, March, 29-38.

Wortley, R., \& Mazerolle, L. G. (2008). Environmental criminology and crime analysis: situating the theory, analytic approach and application. In R. Wortley, \& L. G. Mazerolle (Eds.), Environmental criminology and crime analysis (pp. 118). Cullompton: Willan.

Xu, Y., Fiedler, M. L., \& Flaming, K. H. (2005). Discovering the impact of community policing: the broken windows, collective efficacy, and citizens judgment. Journal of Research in Crime and Delinquency, 42(2), 147-186. http://dx.doi.org/10.1177/0022427804266544.

Yang, X. (2006). Exploring the influence of environmental features on residential burglary using spatial-temporal pattern analysis (PhD thesis). Florida, University of Florida.

Ycaza, C. (1992). Crime rate drops in shores. Miami: Journal The Miami Herald.

Zanotto, K. R. (2002). Segurança em área urbana central: configuração, forma urbana e usuários. Porto Alegre: Programa de Pós-Graduação em Planejamento Urbano e Regional, Universidade Federal do Rio Grande do Sul.

Editor: Rodrigo Firmino

Recebido: Jan. 31, 2018

Aprovado: Jul. 23, 2018 